\title{
ANY BEHAVIOUR OF THE MITCHELL ORDERING OF NORMAL MEASURES IS POSSIBLE
}

\author{
JIŘÍ WITZANY \\ (Communicated by Andreas R. Blass)
}

\begin{abstract}
Let $U_{0}, U_{1}$ be two normal measures on $\kappa$. We say that $U_{0}$ is in the Mitchell ordering less than $U_{1}, U_{0} \triangleleft U_{1}$, if $U_{0} \in \operatorname{Ult}\left(V, U_{1}\right)$. The relation is well-known to be transitive and well-founded. It has been an open problem to find a model where $\triangleleft$ embeds the four-element poset ? ?. We find a generic extension where all well-founded posets are embeddable. Hence there is no structural restriction on the Mitchell ordering. Moreover we show that it is possible to have two $\triangleleft$-incomparable measures that extend in a generic extension into two $\triangleleft$-comparable measures.
\end{abstract}

We address the question of possible behaviours of the Mitchell ordering of normal measures.

In the well-known Mitchell's model $L[\overrightarrow{\mathcal{U}}]$ the ordering of measures on a cardinal $\kappa$ is linear [Mi83]. S. Baldwin constructed a model where $\triangleleft$ is a pre-well-ordering [Ba85] (a well-founded poset is pre-well-ordered iff $\forall p, q \in P: p<_{P} q$ iff $o_{P}(p)<$ $o_{P}(q)$ where $o_{P}(p)$ is the rank of $p$ in $P$ ). Recently J. Cummings [Cu93] described the Mitchell ordering in a particular generic extension where it embeds any wellfounded poset that does not embed the four-element poset:

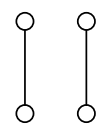

We say that a well-founded poset $P$ embeds into the Mitchell ordering of normal measures on $\kappa$ if there are different measures $\left\{U_{p} ; p \in P\right\}$ on $\kappa$ so that $U_{p} \triangleleft U_{q}$ iff $p<_{P} q$. I show that there is a generic extension where any well-founded poset (with certain cardinality restrictions) embeds into $\triangleleft$. It proves that there is no structural restriction on the Mitchell ordering. However it still remains open to construct a model where all measures on $\kappa$ ordered by the Mitchell ordering are isomorphic to a given poset, e.g. there are only four measures ordered according to the figure above. That would certainly need to go into inner models, possibly

Received by the editors February 11, 1994 and, in revised form, July 18, 1994; some results of this paper were presented at the annual meeting of the Association for Symbolic Logic at the University of Florida, March 5-8, 1994.

1991 Mathematics Subject Classification. Primary 03E35, 03E55.

Key words and phrases. Stationary sets, reflection, measurable cardinals, repeat points. 
first starting with a generic extension. S. Baldwin in [Ba85] notes that his method hopelessly fails especially for posets that embed the four-element poset.

We say that a function $f: \kappa \rightarrow V_{\kappa}$ is a Laver's function on $\kappa$ if

$$
\forall x \in \mathcal{P}\left(\kappa^{+}\right) \exists U \text { a measure on } \kappa:\left(j_{U} f\right)(\kappa)=x
$$

where $j_{U}: V \rightarrow M_{U}=\operatorname{Ult}(V, U)$ is the canonical ultraproduct embedding. It is proved in [W94a] that there is a Laver's function on $\kappa$ if $V=L[\overrightarrow{\mathcal{U}}]$ and $o^{\mathcal{U}}(\kappa)=$ $\kappa^{++}$. It follows from the fact that the measures on $\kappa$ cover $\mathcal{P}\left(\kappa^{+}\right)$in the following sense

$$
\forall x \in \mathcal{P}\left(\kappa^{+}\right) \exists \alpha<\kappa^{++}: x \in \operatorname{Ult}\left(V, U_{\alpha}^{\kappa}\right) .
$$

Theorem 1. Assume that $V=L[\overrightarrow{\mathcal{U}}], o^{\mathcal{U}}(\kappa)=\kappa^{++}$. Then there is a generic extension $V[G]$ preserving cardinalities, cofinalities, and $G C H$ such that any wellfounded poset in $V[G]$ of cardinality $\leq \kappa^{+}$embeds into $\triangleleft_{\kappa}^{V[G]}$. Moreover there is still a Laver's function on $\kappa$.

Let me first state a few well-known facts (see e.g. [Cu93] or [W94a]).

Fact 1. Let $U \triangleleft W$ be two measures on $\kappa, j_{U}: V \rightarrow M_{U}, j_{W}: V \rightarrow M_{W}$, and $j_{U}^{M_{W}}: M_{W} \rightarrow M_{U}^{\prime}=\operatorname{Ult}\left(M_{W}, U\right)$. Then $j_{U}^{M_{W}}=j_{U} \uparrow M_{W}$ and $V_{j_{U}(\kappa)+1} \cap M_{U}^{\prime}=$ $V_{j_{U}(\kappa)+1} \cap M_{U}$.

Fact 2. Let $M, N$ be two inner models of $Z F C, M \subseteq N, N \cap{ }^{\kappa} M \subseteq M$. Let $P \in M$ be a forcing notion such that $N \models$ "P is $\kappa^{+}$-c.c. or $P$ is $\kappa$-closed", and let $G$ be $P$-generic over $N$. Then $N[G] \cap{ }^{\kappa} M[G] \subseteq M[G]$.

Fact 3 (Easton's lemma). Let a notion of forcing $P$ be $\kappa$-c.c., and let $Q$ be $<\kappa$ closed. Then

(i) $Q \Vdash$ "Г̃ is $\check{\kappa}$-c.c.",

(ii) $P \Vdash$ "Q $Q$ is $<\check{\kappa}$-distributive",

(iii) Generics over $V$ for $P$ and $Q$ are mutually generic.

Let $\operatorname{Add}(\alpha, \kappa)$ denote the Cohen forcing adding $\alpha$ Cohen subsets of $\kappa$ : a condition $p \in \operatorname{Add}(\alpha, \kappa)$ is a function $p: \operatorname{dom}(p) \rightarrow\{0,1\}$ such that $\operatorname{dom}(p) \subseteq \alpha \times \kappa$, $|\operatorname{dom}(p)|<\kappa, p<q$ iff $p \supseteq q$. If $\kappa$ is a regular cardinal, then $\operatorname{Add}(\alpha, \kappa)$ is $\kappa^{+}$-c.c. and $<\kappa$-closed. An $\operatorname{Add}(\alpha, \kappa)$-generic filter $G$ is best represented by a subset of $\alpha \times \kappa$. If $A \subseteq \alpha$, then $G \cap(A \times \kappa)$ naturally gives an $\operatorname{Add}($ o.t. $(A), \kappa)$-generic filter. Any bijection $f: \alpha \times \kappa \rightarrow \beta \times \kappa$ gives an isomorphism of $\operatorname{Add}(\alpha, \kappa)$ and $\operatorname{Add}(\beta, \kappa)$; if $G \subseteq \alpha \times \kappa$ is $\operatorname{Add}(\alpha, \kappa)$-generic, then $f[G]$ is $\operatorname{Add}(\beta, \kappa)$-generic.

Proof of Theorem 1. Let $P_{\kappa}$ be an Easton product of $\left\langle\operatorname{Add}\left(1, \lambda^{+}\right) ; \lambda \in \operatorname{Reg}(\kappa)\right\rangle$; we use the trivial forcing if $\lambda<\kappa$ is not a regular cardinal. Since $P_{\kappa}$ is a direct limit, it can be considered to be a subset of $V_{\kappa}$. For regular $\lambda$ factor $P_{\kappa}$ as $P_{\lambda} \times P_{\lambda, \kappa}$; then $P_{\lambda}$ is $\lambda^{+}$-c.c. and $P_{\lambda, \kappa}$ is $\lambda$-closed. Consequently by Easton's Lemma

$$
P_{\lambda} \Vdash " \check{P}_{\lambda, \kappa} \text { is } \check{\lambda} \text {-distributive". }
$$

It follows that $P_{\kappa}$ preserves cardinalities, cofinalities, and GCH. As a matter of fact $P_{\kappa}$ is the well-known Kunen-Paris forcing [KuP71]. Let $G$ be $P_{\kappa}$-generic over $V$.

Assume $P \in V[G]$ is a given well-founded poset of cardinality $\leq \kappa^{+}$. I claim that $P$ can be embedded into $\triangleleft_{\kappa}^{V[G]}$. 
Claim 1. Let $U_{0} \in V$ be a measure on $\kappa, j_{0}: V \rightarrow \operatorname{Ult}\left(V, U_{0}\right)=M_{0}$. Then $j_{0}$ can be lifted to an elementary embedding $j_{0}^{*}: V[G] \rightarrow M_{0}\left[G \times \tilde{H}_{0}\right], j_{0}^{*}(G)=G \times \tilde{H}_{0}$, uniquely determined by $\tilde{H}_{0} \in V[G]$.

Proof. Factor $j_{0}\left(P_{\kappa}\right)$ as $P_{\kappa} \times R$ where $R=\left(j_{0} P_{\kappa}\right)_{\kappa, j_{0} \kappa}$. Since $P_{\kappa}$ is a direct limit, it is enough to find an $R$-generic filter $\tilde{H}_{0} \in V$ over $M_{0}$. By Easton's Lemma $\tilde{H}_{0}$ is then $R$-generic over $M_{0}[G]$. The number of $R$-antichains in $M_{0}$ is $j_{0}\left(\kappa^{+}\right)$, hence the number of these antichains computed in $V$ is only $\kappa^{+}$. Moreover $R$ is $\kappa$-closed in $M_{0}$, thus in $V$, enabling us to build up an $\tilde{H}_{0} \in V$.

The embedding $j_{0}^{*}$ defines a measure $U_{0}^{*} \in V[G]$ extending $U_{0}$; it is easy to see that $j_{0}^{*}$ is the ultraproduct embedding given by $U_{0}^{*}: M_{0}=\left\{\left(j_{0} g\right)(\kappa) ; g \in{ }^{\kappa} V \cap V\right\}$, and it is enough to prove that $M_{0}\left[G \times \tilde{H}_{0}\right]=\left\{\left(j_{0}^{*} g\right)(\kappa) ; g \in{ }^{\kappa} V[G] \cap V[G]\right\}$. Let $x=i_{G \times \tilde{H}_{0}}(\dot{x}) \in M_{0}\left[G \times \tilde{H}_{0}\right]$, find $g \in{ }^{\kappa} V \cap V$ such that $\left(j_{0} g\right)(\kappa)=\dot{x}$, and define $g^{*}(\alpha)=i_{G}(g(\alpha))$ if $g(\alpha)$ as a $P_{\kappa}$-name, then $g^{*} \in{ }^{\kappa} V[G] \cap V[G]$ and $\left(j_{0}^{*} g^{*}\right)(\kappa)=x$.

We consider only one-step extensions of this type. Notice that the forcing $R$ defined above is a product of forcings that always starts with $\operatorname{Add}\left(1, \kappa^{+}\right)^{V}$. Thus we can always factor $\tilde{H}_{0}$ as $H_{0}^{\kappa} \times H_{0}$ where $H_{0}^{\kappa}$ is $\operatorname{Add}\left(1, \kappa^{+}\right)$-generic over $M_{0}[G]$. Now we need to find some sufficient and necessary conditions for $\triangleleft$ on those extensions.

Claim 2. Let $U_{0}^{*}, U_{1}^{*}$ be extensions of $U_{0}, U_{1}$ given by $H_{0}^{\kappa} \times H_{0}$ and $H_{1}^{\kappa} \times H_{1}$. If $U_{0}^{*} \triangleleft U_{1}^{*}$, then $U_{1} \neq U_{0}, U_{1} \Varangle U_{0}$, and $H_{0}^{\kappa} \times H_{0} \in M_{1}\left[G \times H_{1}^{\kappa}\right]$. On the other hand if $U_{0} \triangleleft U_{1}$ and $H_{0}^{\kappa} \times H_{0} \in M_{1}\left[G \times H_{1}^{\kappa}\right]$, then $U_{0}^{*} \triangleleft U_{1}^{*}$.

Proof. Assume $U_{0} \triangleleft U_{1}$ and $H_{0}^{\kappa} \times H_{0} \in M_{1}\left[G \times H_{1}^{\kappa}\right]$. Extend $j_{0}\left\lceil M_{1}=j_{0}^{\prime}\right.$ : $M_{1} \rightarrow M_{0}^{1}=\operatorname{Ult}\left(M_{1}, U_{0}\right)$ to $\tilde{\jmath}_{0}: M_{1}[G] \rightarrow M_{0}^{1}\left[G \times H_{0}^{\kappa} \times H_{0}\right]$ in $M_{1}\left[G \times H_{1}^{\kappa}\right]$. Then $\tilde{\jmath}_{0}=j_{0}^{*}\left\lceil M_{1}[G]\right.$ defines the measure $U_{0}^{*}$ in $M_{1}\left[G \times H_{1}^{\kappa}\right]$ because subsets of $\kappa$ are the same in $V[G]$ and $M_{1}[G]$.

Assume that $U_{0}^{*} \triangleleft U_{1}^{*}$; then $j_{0}(\kappa)<j_{1}(\kappa)$, and so $U_{1}$ cannot be less than $U_{0}$ in the Mitchell ordering. $U_{0}^{*} \in M_{1}\left[G \times H_{1}^{\kappa}\right]$ since the rest of the forcing $\left(j_{0} P_{\kappa}\right)_{\kappa+1, j_{0} \kappa}$ is sufficiently closed. Consequently $j_{0}^{*}(G)=G \times H_{0}^{\kappa} \times H_{0} \in M_{1}\left[G \times H_{1}^{\kappa}\right]$.

Let $P=\left(\Theta,<_{P}\right), \Theta<\kappa^{++}$, so that the ordering of ordinals extends $<_{P}$. Using the $\kappa$-c.c. of $P_{\kappa}$ find a $P_{\kappa}$-name $\dot{P} \subseteq \Theta \times \Theta \times P_{\kappa}$ for $P$ of cardinality $\leq \kappa^{+}$. Since the measures on $\kappa$ cover $\mathcal{P}\left(\kappa^{+}\right)$, there is a $\beta<\kappa^{++}$so that $\dot{P} \in \operatorname{Ult}\left(V, U_{\beta}^{\kappa}\right)$ and $\Theta<\kappa^{++\operatorname{Ult}\left(V, U_{\beta}^{\kappa}\right)}$. Fix a sequence of measures $U_{0} \triangleleft U_{1} \triangleleft \cdots \triangleleft U_{\alpha} \triangleleft \cdots$ $(\alpha<o(P))$ starting with $U_{0}=U_{\beta}^{\kappa}$. Denote $j_{\alpha}: V \rightarrow \operatorname{Ult}\left(V, U_{\alpha}\right)=M_{\alpha}$; then $P \in M_{\alpha}[G]$ and $\Theta<\kappa^{++M_{\alpha}}$ for all $\alpha<o(P)$ by the choice of $U_{0}$ and Fact 1. I am going to find $U_{p}^{*} \in V[G]$ (for $p \in P$ ) extending $U_{o_{P}(p)}$ so that $U_{p}^{*} \triangleleft U_{q}^{*}$ iff $p<_{P} q$.

Claim 3. There is $\tilde{H} \in V[G]$ simultaneously $\operatorname{Add}\left(1, \kappa^{+}\right)$-generic over all $M_{\alpha}[G]$ for $\alpha<o(P)$.

Proof. The number of $\operatorname{Add}\left(1, \kappa^{+}\right)$-antichains in $M_{\alpha}[G]$ computed in $V[G]$ is $\kappa^{+}$for a fixed $\alpha$, hence still $\kappa^{+}$for all $\alpha<o(P)<\kappa^{++}$together. $\operatorname{Add}\left(1, \kappa^{+}\right)$is $\kappa$-closed; thus the generic $\tilde{H}$ can be constructed in $V[G]$.

Let $\pi: 1 \times \kappa^{+} \rightarrow \Theta \times \kappa^{+}$be a bijection in $M_{0}$. Then $\pi[\tilde{H}]$ is $\operatorname{Add}\left(\Theta, \kappa^{+}\right)$-generic over all $M_{\alpha}[G]$. For $q \in P$ let

$$
H_{q}^{\kappa}=\pi_{q}^{-1}\left[\pi[\tilde{H}] \cap\left(\left\{p ; p \leq_{P} q\right\} \times \kappa^{+}\right)\right]
$$


where $\pi_{q}: 1 \times \kappa^{+} \rightarrow\left\{p ; p \leq_{P} q\right\} \times \kappa^{+}$is a bijection in $M_{0}[G]$ (and so in all $\left.M_{\alpha}[G]\right) . H_{q}^{\kappa}$ is obviously $\operatorname{Add}\left(1, \kappa^{+}\right)$-generic over all $M_{\alpha}[G]$. Moreover for $p \in P$ let $g_{p}$ denote the $p$-th $\operatorname{Add}\left(1, \kappa^{+}\right)$-generic of $\pi[\tilde{H}]$,

$$
g_{p}=\{\alpha<\kappa ;(p, \alpha) \in \pi[\tilde{H}]\} .
$$

If $p \leq_{P} q$, then obviously $g_{p} \in M_{\alpha}\left[G \times H_{q}^{\kappa}\right]$ and, on the other hand, if $p \not_{P} q$, then $g_{p}$ is $\operatorname{Add}\left(1, \kappa^{+}\right)$-generic over $M_{\alpha}\left[G \times H_{q}^{\kappa}\right]$, and so $g_{p} \notin M_{\alpha}\left[G \times H_{q}^{\kappa}\right]$. To complete the definition of $U_{q}^{*}$ we need to find an appropriate $\left(j_{\alpha} P_{\kappa}\right)_{\kappa+1, j_{\alpha} \kappa}$-generic filter over $M_{\alpha}$, where $\alpha=o_{P}(q)$. Consider $j_{\alpha}^{\prime}: M_{\alpha+1} \rightarrow \operatorname{Ult}\left(M_{\alpha+1}, U_{\alpha}\right)=M_{\alpha}^{\prime}$, and find an $H_{q} \in M_{\alpha+1}$ which is $\left(j_{\alpha} P_{\kappa}\right)_{\kappa+1, j_{\alpha} \kappa}$-generic over $M_{\alpha}^{\prime}$ as in Claim 1. By Fact $1 V_{j_{\alpha}(\kappa)+1}^{M_{\alpha}}=V_{j_{\alpha}(\kappa)+1}^{M_{\alpha}^{\prime}}$, thus $H_{q}$ is generic over $M_{\alpha}$ as well. $G \times H_{q}^{\kappa} \times H_{q}$ is $j_{\alpha} P_{\kappa}$-generic over $M_{\alpha}$, defining an extension of $j_{\alpha}$

$$
j_{\alpha}^{*}: V[G] \rightarrow M_{\alpha}\left[G \times H_{q}^{\kappa} \times H_{q}\right] .
$$

The embedding $j_{\alpha}^{*}$ defines a measure $U_{q}^{*} \in V[G]$ extending $U_{\alpha} ; U_{q}^{*}$ is actually an element of $M_{\alpha+1}\left[G \times H_{q}^{\kappa}\right]$.

Claim 4. $U_{p}^{*} \triangleleft U_{q}^{*}$ iff $p<_{P} q$.

Proof. Let $U_{p}^{*} \triangleleft U_{q}^{*}$, then by Claim $2 \alpha=o_{P}(p)$ must be strictly less than $\beta=$ $o_{P}(q)$, and $H_{p}^{\kappa} \times H_{p} \in M_{\beta}\left[G \times H_{q}^{\kappa}\right]$. Since $g_{p}$ can be decoded from $H_{p}^{\kappa}$ using $\pi_{p} \in M_{\beta}[G]$, it is in $M_{\beta}\left[G \times H_{q}^{\kappa}\right]$, which is possible only if $p \leq_{P} q$, i.e. $p<_{P} q$.

Let $p<_{P} q$; then $\alpha=o_{P}(p)<o_{P}(q)=\beta$. According to Claim 2 all we need to prove is that $H_{p}^{\kappa} \times H_{p} \in M_{\beta}\left[G \times H_{q}^{\kappa}\right]$. The mappings $\pi_{p}, \pi_{q}$ are both in $M_{\beta}[G]$. Consequently we can compute

$$
H_{p}^{\kappa}=\pi_{p}^{-1}\left[\pi_{q}\left[H_{q}^{\kappa}\right] \cap\left(\left\{p^{\prime} ; p^{\prime} \leq_{P} p\right\} \times \kappa^{+}\right)\right]
$$

in $M_{\beta}\left[G \times H_{q}^{\kappa}\right]$. By the construction $H_{p}$ is in $M_{\alpha+1}$, and so in $M_{\beta}$ by Fact 1 .

Let us prove that there is a Laver's function on $\kappa$ in $V[G]$.

First find a sequence of bijections $\pi_{\lambda}: P_{\lambda} \rightarrow \lambda(\lambda \leq \kappa$ inaccessible) coherent in the following sense:

$$
\forall \lambda \leq \kappa \forall \alpha<o^{\mathcal{U}}(\lambda): j_{U_{\alpha}^{\lambda}}\left(\left\langle\pi_{\lambda^{\prime}} ; \lambda^{\prime}<\lambda\right\rangle\right)(\lambda)=\pi_{\lambda} .
$$

Assume $\pi_{\lambda^{\prime}}$ has been defined for $\lambda^{\prime}<\lambda$. If $o^{\mathcal{U}}(\lambda)=0$ pick any bijection $\pi_{\lambda}$ : $P_{\lambda} \rightarrow \lambda$. If $o^{\mathcal{U}}(\lambda)>0$, then $\pi_{\lambda}$ can be defined as $j_{U_{0}^{\lambda}}\left(\left\langle\pi_{\lambda^{\prime}} ; \lambda^{\prime}<\lambda\right\rangle\right)(\lambda)$ since $j_{U_{0}^{\lambda}}\left(\left\langle P_{\lambda^{\prime}} ; \lambda^{\prime}<\lambda\right\rangle\right)(\lambda)=P_{\lambda}$. Condition (1) is then satisfied because $\overrightarrow{\mathcal{U}}$ forms a coherent sequence of measures. Notice that if $\sigma_{\lambda}$ is the bijection $\lambda^{+} \times \lambda \rightarrow \lambda^{+}$ given by the maximolexicographical ordering of $\lambda^{+} \times \lambda$, then also

$$
j_{U_{\alpha}^{\lambda}}\left(\left\langle\sigma_{\lambda^{\prime}} ; \lambda^{\prime}<\lambda\right\rangle\right)(\lambda)=\sigma_{\lambda} \quad \text { for any } \alpha<o^{\mathcal{U}}(\lambda) .
$$

A $P_{\lambda}$-name $\dot{x} \subseteq \lambda^{+} \times P_{\lambda}$ for a subset of $\lambda^{+}$can be uniquely coded using $\pi_{\lambda}$ and $\sigma_{\lambda}$ as a subset of $\lambda^{+}$. If $f(\lambda)$ codes in this way a $P_{\lambda}$-name $\dot{x}$ for a subset of $\lambda^{+}$ define $f^{*}(\lambda)=i_{G \uparrow P_{\lambda}}(\dot{x})$. It is easy to verify that this defines a Laver's function on $\kappa$ in $V[G]$. Theorem 1 is proved.

We can still ask what well-founded $\kappa^{++}$-like posets can be embedded into $\triangleleft$. For example, can we embed the poset consisting of a chain of length $\kappa^{++}$and one incomparable element? The answer is positive; however, in this case we have to destroy the covering of $\mathcal{P}\left(\kappa^{+}\right)$. 
Theorem 2. Assume that $V=L[\overrightarrow{\mathcal{U}}], o^{\mathcal{U}}(\kappa)=\kappa^{++}$. Then there is a generic extension $V[G]$ preserving cardinalities, cofinalities, and $G C H$ such that all wellfounded $\kappa^{++}$-like posets in $V$ and all well-founded posets of cardinality $\leq \kappa^{+}$in $V[G]$ are embeddable into $\triangleleft_{\kappa}^{V[G]}$.

Proof. Let $G=H \times \tilde{G}$ be $\operatorname{Add}\left(\kappa^{++}, \kappa^{+}\right) \times P_{\kappa}$-generic over $V$ where $P_{\kappa}$ is the Kunen-Paris forcing. Cardinalities, cofinalities, and GCH are obviously preserved in $V[G]$.

If $U_{0} \in V$ is a measure on $\kappa, j_{0}: V \rightarrow \operatorname{Ult}\left(V, U_{0}\right)=M_{0}$, and $j_{0}^{*}: V[\tilde{G}] \rightarrow$ $M_{0}\left[\tilde{G} \times H_{0}^{\kappa} \times H_{0}\right]$ an extension of $j_{0}$ defined in $V[\tilde{G} \times H]$, then $U_{0}^{*}=\{X \in V[\tilde{G}] ; X \subseteq$ $\kappa$ and $\left.\kappa \in j_{0}^{*}(X)\right\}$ is a measure in $V[\tilde{G} \times H]$ because $H$ does not add any new subsets of $\kappa$. Similarly as in the proof of Theorem 1 if $U_{0}^{*}, U_{1}^{*}$ are two such extensions of $U_{0}, U_{1}$ given by $H_{0}^{\kappa} \times H_{0}, H_{1}^{\kappa} \times H_{1}$, then $U_{0}^{*} \triangleleft U_{1}^{*}$ implies $U_{1} \neq U_{0}, U_{1} \Varangle U_{0}$, $H_{0}^{\kappa} \times H_{0} \in M_{1}\left[G \times H_{1}^{\kappa}\right]$. On the other hand $U_{0} \triangleleft U_{1}, H_{0}^{\kappa} \times H_{0} \in M_{1}\left[G \times H_{1}^{\kappa}\right]$ implies $U_{1}^{*} \triangleleft U_{1}^{*}$.

Let $P \in V$ be a $\kappa^{++}$-like poset.

Claim. $P$ can be enumerated as $\left\langle p_{\alpha} ; \alpha<\kappa^{++}\right\rangle$so that $p_{\alpha}<_{P} p_{\beta}$ implies $\alpha<\beta$. Consequently there is a sequence of measures $U_{0} \triangleleft \cdots \triangleleft U_{\alpha} \triangleleft \cdots\left(\alpha<\kappa^{++}\right)$on $\kappa$ so that

$$
\forall \alpha<\kappa^{++}: M_{\alpha} \models "|\alpha| \leq \kappa^{+} " \text { and }\left\{\gamma ; p_{\gamma} \leq_{P} p_{\alpha}\right\} \in M_{\alpha},
$$

where $j_{\alpha}: V \rightarrow M_{\alpha}=\operatorname{Ult}\left(V, U_{\alpha}\right)$.

Proof. Define the enumeration by induction: Start with any well-ordering $\prec$ of $P$ of order type $\kappa^{++}$. Assume that $\left\langle p_{\alpha} ; \alpha<\mu\right\rangle$ has been defined so that

$$
\forall \alpha<\mu:\left\{q \in P ; q<_{P} p_{\alpha}\right\} \subseteq\left\{p_{\gamma} ; \gamma<\alpha\right\} .
$$

Let $p_{\mu}$ be the $\prec$-first element of $P \backslash\left\{p_{\alpha} ; \alpha<\mu\right\}$ that is minimal with respect to $<_{P}$. Then the sequence $\left\langle p_{\alpha} ; \alpha<\kappa^{++}\right\rangle$clearly exhausts all elements of $P$ : Assume that $\left\{p_{\alpha} ; \alpha<\kappa^{++}\right\} \subsetneq P$; let $p \in P \backslash\left\{p_{\alpha} ; \alpha<\kappa^{++}\right\}$be a $<_{P}$-minimal element. Find $\mu<\kappa^{++}$such that

$$
\left\{q \in P ; q<{ }_{P} p\right\} \cup\left\{p_{\alpha} ; \alpha<\kappa^{++} \& p_{\alpha} \prec p\right\} \subseteq\left\{p_{\alpha} ; \alpha<\mu\right\} .
$$

Then $p$ is still $<_{P}$-minimal in $P \backslash\left\{p_{\alpha} ; \alpha<\mu\right\}$ and so $p_{\mu} \preceq p$-a contradiction.

The second part of the claim follows easily from the fact that the measures on $\kappa$ cover $\mathcal{P}\left(\kappa^{+}\right)$.

Define $U_{p_{\alpha}}^{*} \in V[G]$ extending $U_{\alpha}$ as follows: Put

$$
H_{\alpha}^{\kappa}=\pi_{\alpha}\left[H \cap\left(\left\{\gamma ; p_{\gamma} \leq_{P} p_{\alpha}\right\} \times \kappa^{+}\right)\right]
$$

where $\pi_{\alpha}:\left\{\gamma ; p_{\gamma} \leq_{P} p_{\alpha}\right\} \times \kappa^{+} \rightarrow 1 \times \kappa^{+}$is a bijection in $M_{\alpha} . H_{\alpha}^{\kappa}$ is $\operatorname{Add}\left(1, \kappa^{+}\right)$generic over $M_{\alpha}[\tilde{G}]$ - the point is that $H$ is now generic over all $M_{\alpha}[\tilde{G}]$ as $\alpha<\kappa^{++}$. Then find an $H_{\alpha} \in M_{\alpha+1}$ that is $\left(j_{\alpha} P_{\kappa}\right)_{\kappa+1, j_{\alpha} \kappa}$-generic over $\operatorname{Ult}\left(M_{\alpha+1}, U_{\alpha}\right)$. That defines a measure $U_{p_{\alpha}}^{*}$ extending $U_{\alpha}$. Similarly as in the proof of Theorem $1 U_{p_{\alpha}}^{*} \triangleleft$ $U_{p_{\beta}}^{*}$ iff $p_{\alpha}<_{P} p_{\beta}$.

Finally let $P \in V[G]$ be a well-founded poset of cardinality $\kappa^{+}$. Then by the $\kappa^{++}$-c.c. of $\operatorname{Add}\left(\kappa^{++}, \kappa^{+}\right)$there is $\vartheta<\kappa^{++}$so that $P \in V[\tilde{G} \times(H \uparrow \vartheta)]$. Represent 
$P$ as $\left([\vartheta, \Theta),<_{P}\right), \vartheta<\Theta<\kappa^{++}$, and find $U_{0} \triangleleft \cdots \triangleleft U_{\alpha} \triangleleft \cdots(\alpha<o(P))$ so that $P \in M_{0}\left[\tilde{G} \times(H\lceil\vartheta)]\right.$ and $\vartheta<\kappa^{++M_{0}}$. Then define $U_{q}^{*}$ extending the $U_{o_{P}(q)}$ setting

$$
H_{q}^{\kappa}=\pi_{q}\left[H \cap\left(\left(\vartheta \cup\left\{p ; p<_{P} q\right\}\right) \times \kappa^{+}\right)\right]
$$

where $\pi_{q}:\left(\vartheta \cup\left\{p ; p<_{P} q\right\}\right) \times \kappa^{+} \rightarrow 1 \times \kappa^{+}$is a bijection in $M_{0}[\tilde{G} \times(H \uparrow \vartheta)]$ such that $\pi_{q} \uparrow\left(\vartheta \times \kappa^{+}\right)$is in $M_{0}$. Find $H_{q}$ as in the proof of Theorem 1. Then similarly as in the proof $H_{p}^{\kappa} \in M_{\beta}\left[\tilde{G} \times H_{q}^{\kappa}\right]$ iff $p<_{P} q$ iff $U_{p}^{*} \triangleleft U_{q}^{*}$.

Remark 1. J. Cummings in [Cu93] starts with $V=K\left[\overrightarrow{\mathcal{U}}_{\max }\right]\left(o^{\mathcal{U}}(\kappa)<\kappa^{++}\right)$, then applies a forcing similar to the Kunen-Paris forcing, and classifies all measures in $V[G]$ using special properties of $K\left[\overrightarrow{\mathcal{U}}_{\text {max }}\right]$. More specifically the measures are divided into blocks of incomparable measures $M(\alpha, \beta)\left(\alpha<\beta \leq o^{\mathcal{U}}(\kappa)\right)$ of cardinality $\kappa^{+}$or $\kappa^{++}$so that for $U \in M(\alpha, \beta), W \in M(\gamma, \delta): U \triangleleft W$ iff $\beta \leq \gamma$.

Starting with $V=K\left[\overrightarrow{\mathcal{U}}_{\text {max }}\right]$ we can use the same method to classify measures in $V[G]$ where $G$ is $P_{\kappa}$-generic over $V$.

A finite normal iteration $j: V \rightarrow N$ of length $n+1$ is an iteration of ultraproducts by measures on $\kappa=\kappa_{0}<\kappa_{1}<\cdots<\kappa_{n}$. Any finite normal iteration $j: V \rightarrow N$ that starts with a measure $U$ gives $\kappa^{++}$extensions $U^{*}$ in $V[G]$ of $U$ such that $j_{U^{*}} \uparrow V=j, j_{U^{*}}(G)=G * H^{\kappa} * H$. All measures in $V[G]$ are produced in this way. We can give sufficient and necessary conditions for $\triangleleft$ in $V[G]$ : Let $U_{0}^{*}, U_{1}^{*}$ extending $U_{0}, U_{1}$ be given by finite normal iterations $j_{0}: V \rightarrow N_{0}, j_{1}: V \rightarrow N_{1}$ and $H_{0}^{\kappa} * H_{0}, H_{1}^{\kappa} * H_{1}$. Then $U_{0}^{*} \triangleleft U_{1}^{*}$ iff $j_{0} \uparrow \operatorname{Ult}\left(V, U_{1}\right)$ is an internal iteration in $\operatorname{Ult}\left(V, U_{1}\right)$ and

$$
H_{0}^{\kappa} * H_{0} \in \operatorname{Ult}\left(V, U_{1}\right)\left[G * H_{1}^{\kappa}\right] .
$$

However, we can hardly describe the Mitchell ordering $\triangleleft$ in $V[G]$ in a simpler manner.

That is illustrated by the following: Let $U_{0}$ be the minimal measure on $\kappa$ in $V$, and let $U_{0}^{*}$ be its one-step extension using $H_{0}^{\kappa} * H_{0} \in V[G]$. We have seen that there may be measures above $U_{0}^{*}$ even if $H_{0}^{\kappa} * H_{0} \notin M_{\alpha}[G]$ for all $\alpha<o(\kappa)$ $\left(M_{\alpha}=\operatorname{Ult}\left(V, U_{\alpha}^{\kappa}\right)\right)$. However it is also possible that there are no measures above $U_{0}^{*}$. It follows from the following joint lemma with J. Zapletal.

Lemma. There is $H_{0}^{\kappa} \in V[G] \operatorname{Add}\left(1, \kappa^{+}\right)$-generic over $M_{0}[G]$ such that for any $\alpha<o^{\mathcal{U}}(\kappa), \alpha>0$, there is no $H_{1}^{\kappa} \in V[G] \operatorname{Add}\left(1, \kappa^{+}\right)$-generic over $M_{\alpha}[G], \alpha<o(\kappa)$, satisfying $H_{0}^{\kappa} \in M_{\alpha}[G]\left[H_{1}^{\kappa}\right]$.

Proof. Let $R \subseteq \kappa^{+} \times \kappa^{+}$be a well-ordering of order type $\gamma$ where $\gamma>\kappa^{++M_{\alpha}}$ for all $\alpha<o(\kappa)<\kappa^{++}$. Notice that if $H_{1}^{\kappa}$ is any $\operatorname{Add}\left(1, \kappa^{+}\right)$-generic over $M_{\alpha}[G]$, then $R \notin M_{\alpha}[G]\left[H_{1}^{\kappa}\right]$; otherwise $\gamma$ would be less than $\kappa^{++M_{\alpha}}$. So it would be enough to code $R$ into $H_{0}^{\kappa}$. Let $\left\langle a_{\alpha} ; \alpha<\kappa^{+}\right\rangle \in M_{1}[G]$ canonically enumerate $\kappa^{+} \times \kappa^{+}$and $\left\langle D_{\alpha} ; \alpha<\kappa^{+}\right\rangle \in M_{1}[G]$ enumerate all $M_{0}[G]$-dense subsets of $\operatorname{Add}\left(1, \kappa^{+}\right)$, each set $D_{\alpha}$ enumerated by ordinals $<\kappa^{+}$. Construct a descending sequence of conditions $\left\langle p_{\alpha} ; \alpha<\kappa^{+}\right\rangle \in V[G]$ as follows: Assume $\left\langle p_{\delta} ; \delta<\alpha\right\rangle$ has been constructed, then find the first $q \in D_{\alpha}$ extending $\bigcup\left\{p_{\delta} ; \delta<\alpha\right\}$, let $\eta=\sup \{\xi+1 ; \xi \in \operatorname{dom}(q)\}$, and put $p\lceil\eta=q$ and

$$
p(\eta)=\left\{\begin{array}{cc}
1 & \text { iff } a_{\alpha} \in R \\
0 & \text { otherwise }
\end{array}\right.
$$


That gives an $\operatorname{Add}\left(1, \kappa^{+}\right)$-generic filter $H_{0}^{\kappa}$ over $M_{0}[G]$ such that for $\alpha>0$

$$
H_{0}^{\kappa} \in M_{\alpha}[G]\left[H_{1}^{\kappa}\right]
$$

implies $R \in M_{\alpha}[G]\left[H_{1}^{\kappa}\right]$.

Remark 2. It is not true in general that $U_{0}^{*} \triangleleft U_{1}^{*}$ implies $U_{0} \triangleleft U_{1}$ as Claim 2 in the proof of Theorem 1 might suggest: There is a model $M$, two measures $U_{0}, U_{1} \in M$ and their extensions $U_{0}^{*}, U_{1}^{*}$ in $M[\tilde{G}]$, where $\tilde{G}$ is $P_{\kappa}$-generic over $M$, so that

$$
U_{0} \rtimes U_{1} \text { but } U_{0}^{*} \triangleleft U_{1}^{*} .
$$

Proof. Start with two measures $U_{0} \triangleleft U_{1}$ in $V$, and with the corresponding canonical embeddings $j_{0}: V \rightarrow M_{0}, j_{1}: V \rightarrow M_{1}$. Let $G \times \tilde{G}$ be $P_{\kappa} \times P_{\kappa}$-generic over $V$. Then find $H_{0}^{\kappa} \times H_{1}^{\kappa} \times H_{2}^{\kappa} \in V$ a filter $\operatorname{Add}\left(3, \kappa^{+}\right)$-generic over $M_{1}, H_{1} \times$ $\tilde{H}_{1} \in V$ a filter $\left(j_{1} P_{\kappa}\right)_{\kappa+1, j_{1} \kappa} \times\left(j_{1} P_{\kappa}\right)_{\kappa+1, j_{1} \kappa}$-generic over $M_{1}$, and $H_{0} \times \tilde{H}_{0} \in$ $M_{1}$ a filter $\left(j_{0} P_{\kappa}\right)_{\kappa+1, j_{0} \kappa} \times\left(j_{0} P_{\kappa}\right)_{\kappa+1, j_{0} \kappa}$-generic over $M_{0}$. Using Easton's Lemma $G, \tilde{G}, H_{0}^{\kappa}, H_{1}^{\kappa}, H_{2}^{\kappa}, H_{1}, \tilde{H}_{1}$ are mutually generic over $M_{1}$ and $G, \tilde{G}, H_{0}^{\kappa}, H_{1}^{\kappa}, H_{2}^{\kappa}, H_{0}$, $\tilde{H}_{0}$ are mutually generic over $M_{0}$. First extend $U_{0}, U_{1}$ to $U_{0}^{*}, U_{1}^{*}$ in $M=V[G]$ so that $j_{1}^{*}(G)=G \times H_{1}^{\kappa} \times H_{1}$ and $j_{0}^{*}(G)=G \times\left(H_{0}^{\kappa} \otimes H_{1}^{\kappa}\right) \times H_{0}$ where $H_{0}^{\kappa} \otimes H_{1}^{\kappa}$ denotes a coding of $H_{0}^{\kappa}, H_{1}^{\kappa}$ into an $\operatorname{Add}\left(1, \kappa^{+}\right)$-generic. Obviously $U_{0}^{*} \nless U_{1}^{*}$ since $H_{0}^{\kappa} \notin M_{1}\left[j_{1}^{*}(G)\right]$. Then extend $U_{0}^{*}, U_{1}^{*}$ to $U_{0}^{* *}, U_{1}^{* *}$ in $M[\tilde{G}]=V[G \times \tilde{G}]$ so that $j_{1}^{* *}(\tilde{G})=\tilde{G} \times\left(H_{0}^{\kappa} \otimes H_{2}^{\kappa}\right) \times \tilde{H}_{1}$ and $j_{0}^{* *}(\tilde{G})=\tilde{G} \times H_{2}^{\kappa} \times \tilde{H}_{0}$. Then $U_{0}^{* *} \triangleleft U_{1}^{* *}$ since $U_{0}^{*}, j_{0}^{* *}(\tilde{G}) \in M_{1}\left[j_{1}^{* *}(G \times \tilde{G})\right]$.

\section{ACKNOWLEDGMENT}

I want to express my gratitude to T. Jech and also to J. Zapletal for many valuable discussions and remarks on the subject.

\section{REFERENCES}

[Ba85] S. Baldwin, The $\triangleleft$-ordering on normal ultrafilters, J. Symbolic Logic 51 (1985), 936-952. MR 87d:03124

[Cu93] J. Cummings, Possible behaviors for the Mitchell ordering, Ann. Pure Appl. Logic (to appear).

[KuP71] K. Kunen and J. B. Paris, Boolean extensions and measurable cardinals, Ann. of Math. Logic 2 (1971), 359-377. MR 43:3114

[Mi83] W.J. Mitchell, Sets constructible from sequences of measures: revisited, J. Symbolic Logic 48 (1983), 600-609. MR 85j:03052

[W94a] J. Witzany, Possible behaviours of the reflection ordering of stationary sets, J. Symbolic Logic (to appear).

[W94b] J. Witzany, Reflection of stationary sets and the Mitchell ordering of normal measures, Ph.D. thesis, Pennsylvania State University, 1994.

Department of Mathematics, The Pennsylvania State University, University Park, Pennsylvania 16802

Current address: Department of Mathematics, University of California, Los Angeles, California 90024-1555

E-mail address: witzany@math.psu.edu

E-mail address: jwitzany@math.ucla.edu 